\title{
Predicción Semana Santa 2017: desde el largo hasta el corto plazo
}

\section{AnTONio García MÉNDEZ}

Ex Área de Técnicas y Aplicaciones de Predicción (ATAP), Agencia Estatal de Meteorología (AEMET)

Ex Meteorological Analyst, European Centre for Medium-range Weather Forecasts-Centro Europeo de Predicción a Plazo Medio- (ECMWF)

¿Es verdad que siempre llueve en Semana Santa?

Título de artículo en elPeriódico, 11-04-2017 - LAURA ESTIRADO

El motivo de este ejercicio de predicción es el de mostrar la utilización de las herramientas disponibles en las páginas web del ECMWF moviéndonos desde la predicción mensual, al medio plazo y finalmente al corto plazo. Todos los productos que componen este ejercicio, provienen de la citada web con excepción de las series de datos de verificación que tienen un tratamiento diferente que se explicará más tarde.

Palabras clave: sistemas de predicción por conjuntos aplicados a la predicción de corto y medio plazo, predicción probabilista, predicción Semana Santa 2017, proceso de predicción en cascada. 

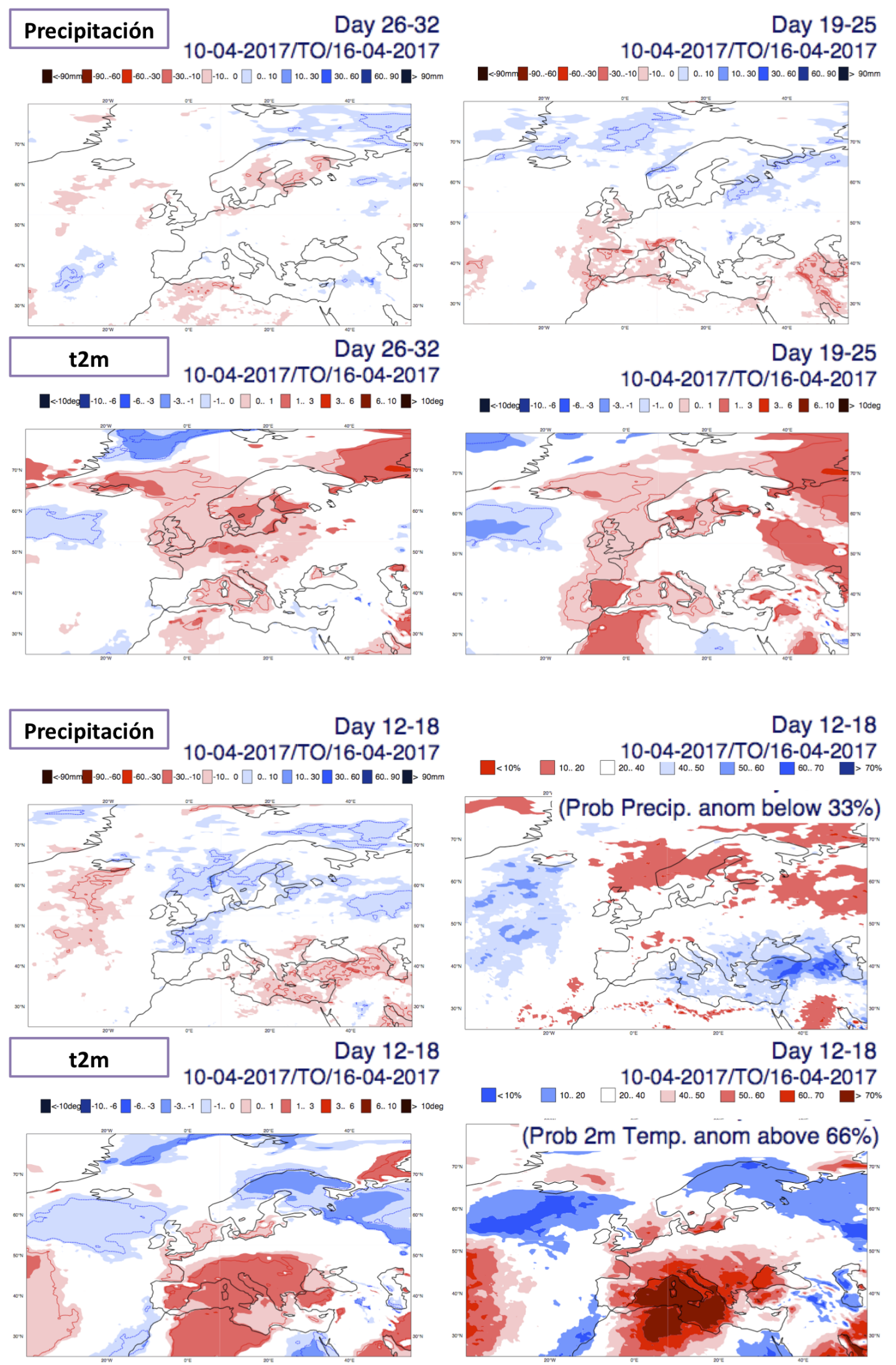

Figura 58.1: Anomalías de precipitación y temperatura a 2 metros (siempre respecto al clima del modelo), 4 y 3 semanas antes (arriba) y 2 y 1 semanas antes (abajo), en la predicción mensual del ECMWF. 


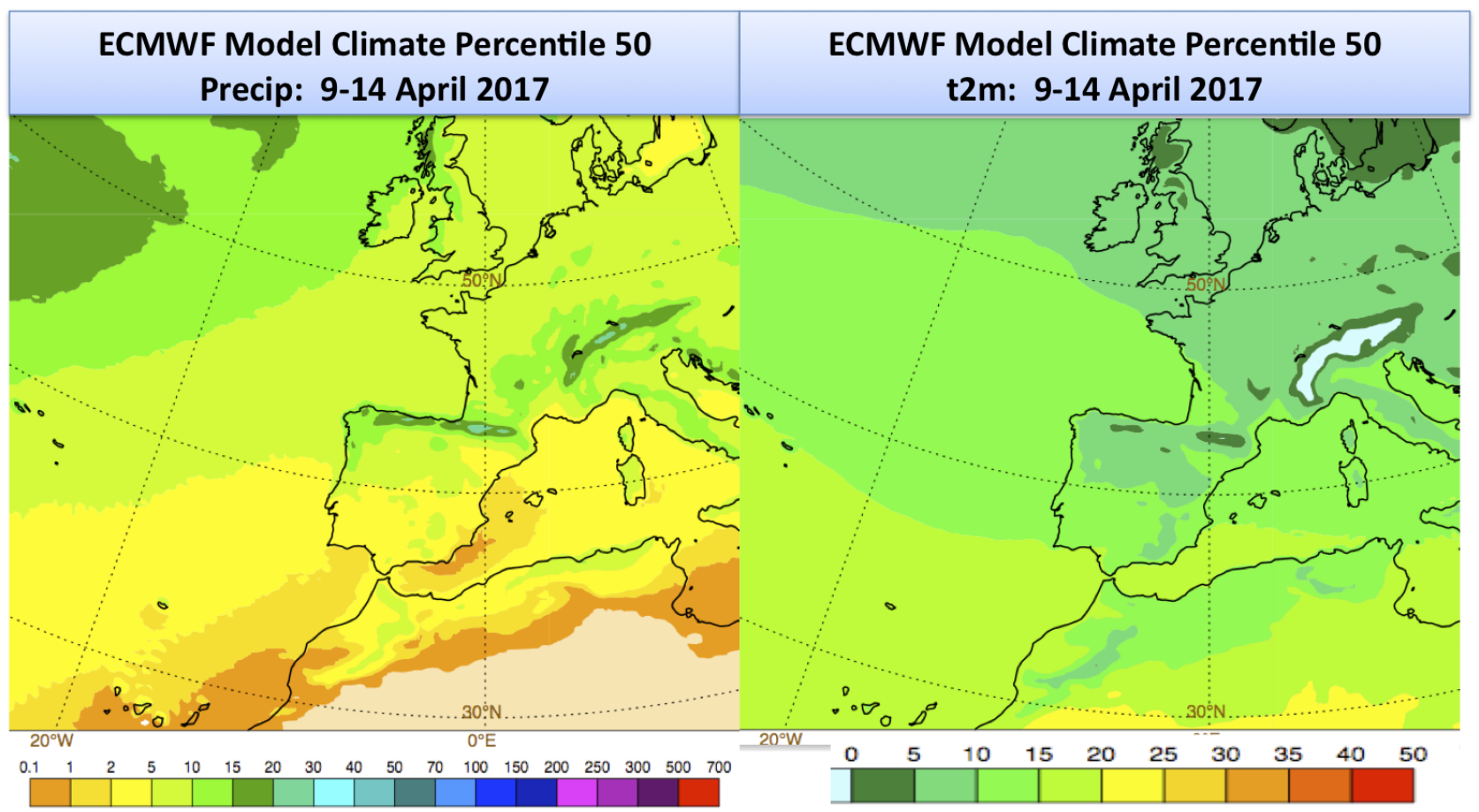

Figura 58.2: Climatología del ECENS, percentil 50, tanto de precipitación como de temperatura a 2 metros para el periodo 9 a 14 de abril de 2017.

\subsection{Predicción mensual}

Un mes antes del comienzo de la Semana Santa comienzo a mirar la predicción mensual del ECMWF [3]. La Figura 58.1 en la página anterior, arriba, muestra las anomalías de precipitación y temperatura a 2 metros (siempre respecto al clima del modelo), 4 y 3 semanas antes. A este plazo solo cabe esperar indicaciones débiles y en muchos casos contradictorias, a no ser que se esperen situaciones con desviaciones grandes respecto a los valores climáticos. De hecho, en la figura para la semana 4 (paneles de la izquierda) solo se aprecian condiciones neutras. Tres semanas antes, aparecen indicaciones débiles de déficit de precipitación y temperaturas por encima de los valores climáticos en el interior peninsular.

Dos semanas antes (Figura 58.1 en la página anterior, abajo), el promedio de la predicción semanal para precipitación, vuelve a mostrar condiciones neutras tanto en términos de anomalías como de probabilidades de anomalías en el tercil inferior del clima. Más consistente parece la temperatura a 2 metros, tanto las anomalías como la probabilidad de estas anomalías de situarse en el tercil superior del clima así lo indican. No hay que olvidar que por regla general, la predecibilidad en términos de temperatura es mayor que para la precipitación. Conviene en este punto mirar la cli- matología del modelo para la semana que es nuestro objetivo.

La Figura 58.2 muestra la climatología del modelo (sección 27.7.1 en la página 419) para el percentil 50 tanto de precipitación como de temperatura a 2 metros para el periodo 9 a 14 de abril de 2017.

\subsection{Consistencia: ¿qué hay que mirar?}

Llegados a este punto, nos enfrentamos a los típicos problemas relacionados con la consistencia de los modelos numéricos para plazos superiores a cinco días. Aquí es donde la operación del ECENS muestra el camino a seguir a los predictores operativos. Antes de la aparición operativa del ECENS, los predictores tendían a utilizar el SPC del «hombre pobre» $[1,2$, 4] (sec. 13.2.2 en la página 168y sec. 34.3). Esto es, mirar distintos modelos, comprobar cualitativamente la dispersión y después o bien utilizar un promedio o decantarse por el modelo en el que el predictor confiase más. En definitiva, todo entreverado por una dosis muy alta de subjetividad y que podía funcionar más o menos bien, dependiendo de las habilidades personales de los predictores. 

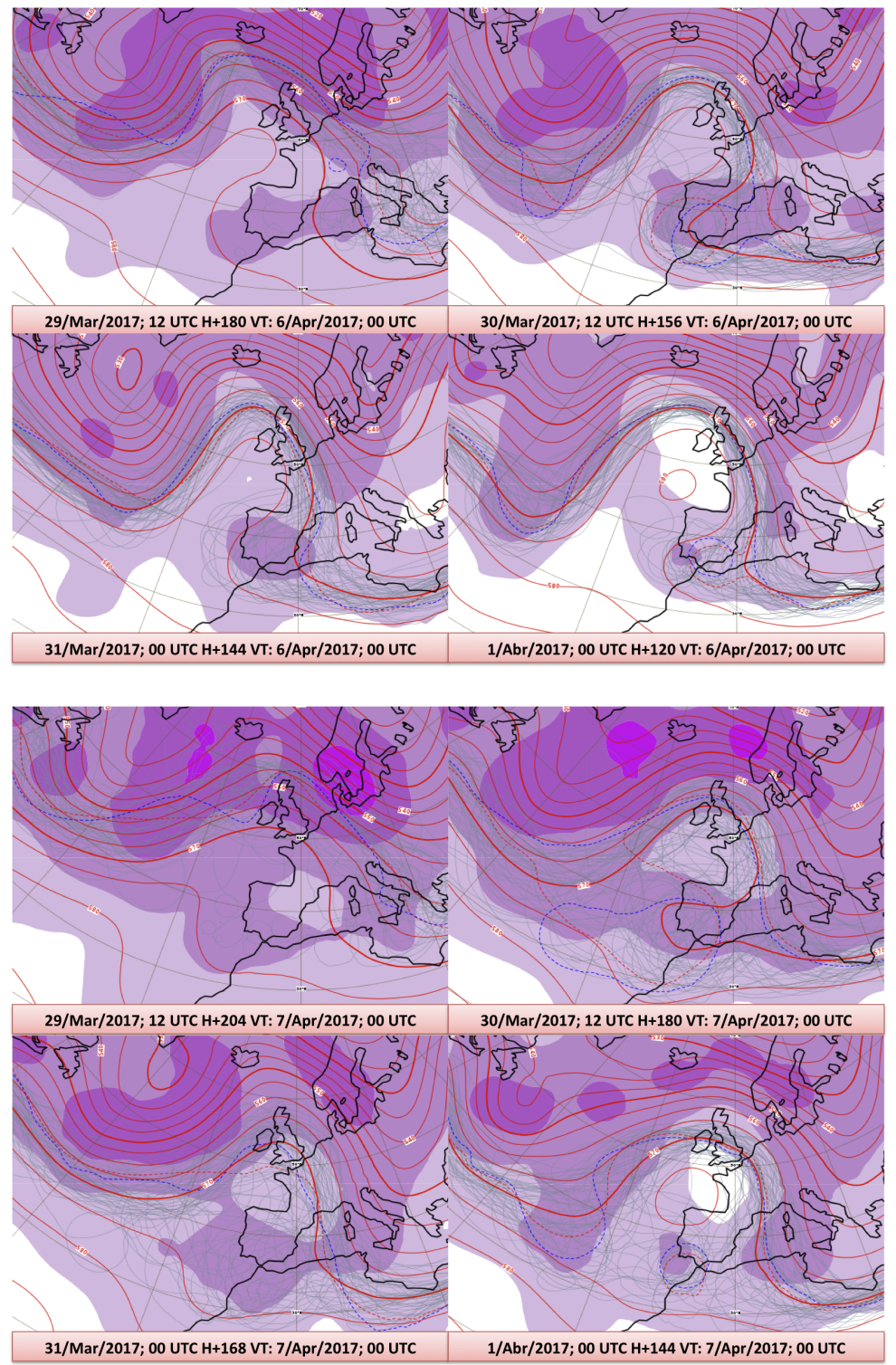

Figura 58.3: Predicciones Z500 del ECENS válidas para 6 y 7 de abril (ver texto).

La Figura 58.3, arriba, muestra predicciones de altura geopotencial a $500 \mathrm{hPa}$ con base en cuatro días consecutivos, 29,30 y 31 de marzo y 1 de abril, todas ellas válidas para el 6 de abril de 2017. Se trata por tanto de predicciones $\mathrm{H}+180, \mathrm{H}+156, \mathrm{H}+144$ y $\mathrm{H}+120$. Los mapas de promedio-dispersión (ver sección 27.5.1 en la página 412) están compuestos por promedio del ensemble (ensemble mean, EM, isolíneas rojas), la dispersión (sombreado) y espaguetis para la isolínea de 570 dam de los miembros del ECENS. El control es la línea rota roja y el modelo determinista la linea rota azul.

Las predicciones deterministas presentan una conducta saltarina sobre el sur peninsular, oscilando entre una vaguada en el Mediterráneo suroccidental y una posible depresión aislada de niveles altos (DANA) sobre el sur de la Península. La media del ECENS presenta una conducta más consistente. La Figura 58.3, abajo, muestra predicciones basadas en los mismos días pero válidas para el 7 de abril de 2017 a 00 UTC $(\mathrm{H}+204, \mathrm{H}+180, \mathrm{H}+168$ y H+144). La conducta oscilante de la predicción determinista sigue siendo clara y también la mayor consistencia del EM. Conviene resaltar aquí que no estamos ante un caso con dispersión elevada. Este tipo de conducta ocurre con mucha frecuencia, por regla general el EM muestra mucha mejor consistencia que las salidas deterministas. Tampoco conviene olvidar que las verificaciones sobre un gran numero de casos en áreas amplias indican que el EM verifica mejor, estadísticamente hablando, que el modelo determinista desde el D+3 en adelante (secs. 15.7 en la página 221 y 13.6.3 en la página 178). 


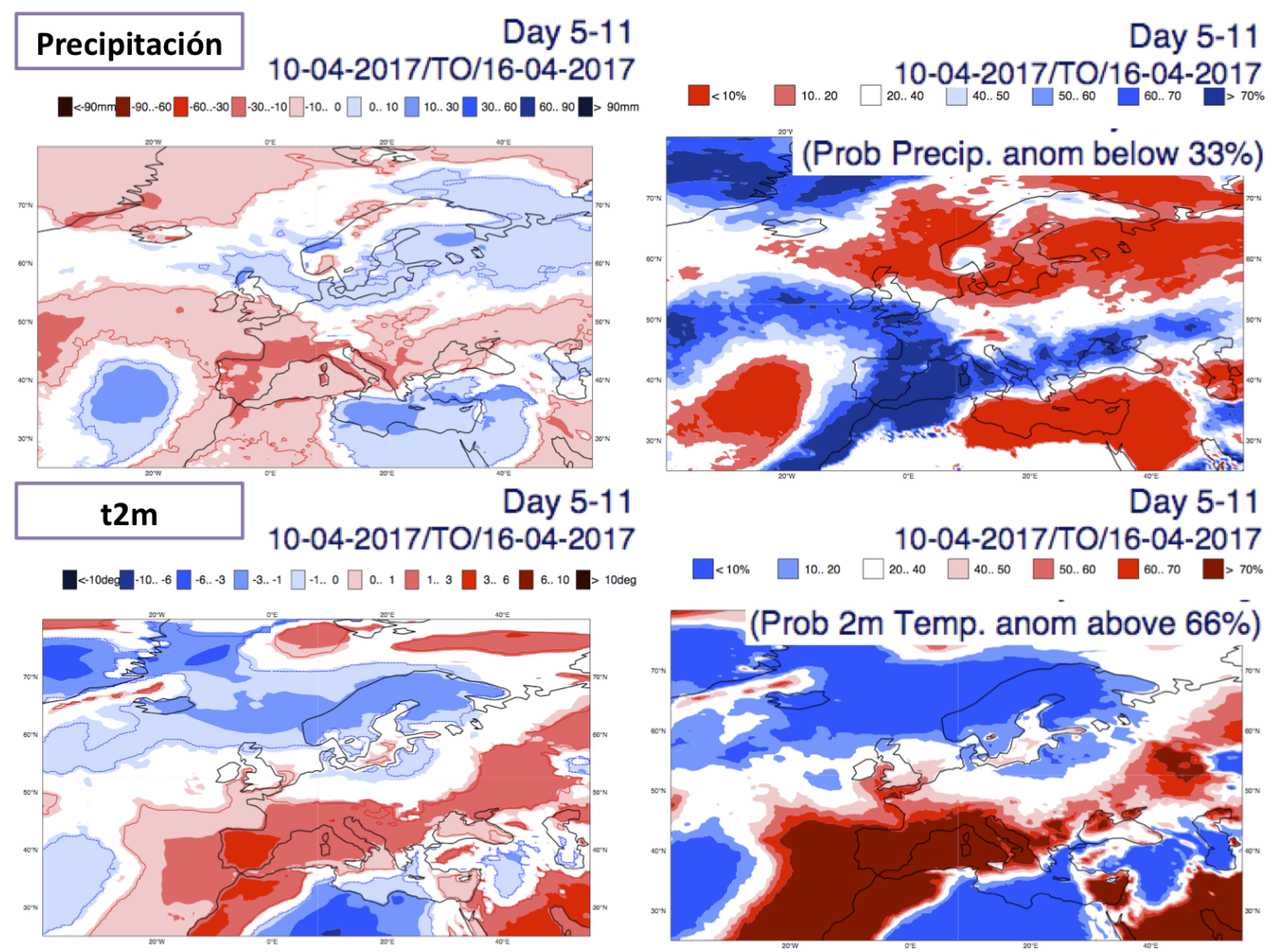

Figura 58.4: Promedios de precipitación y temperatura a 2 metros de la predicción mensual para el periodo 10 a 16 de abril de 2017.

Volviendo a la predicción mensual, la Figura 58.4 muestra los promedios de precipitación y temperatura a 2 metros para el periodo 10 a 16 de abril de 2017. De nuevo estos promedios se presentan aquí en términos de anomalías y probabilidades de que estas anomalías se sitúen en el tercil inferior para la precipitación y en el tercil superior para la temperatura. Lo que se aprecia en esta figura son anomalías secas con alta probabilidad de encontrarse en el tercil inferior del clima para la precipitación y anomalías cálidas con altas probabilidades de encontrarse en el tercil superior del clima para la temperatura.

\subsection{Escenarios, probabilidades, ECENS y corto plazo}

Las Figuras 58.5 y 58.6 en la página siguiente muestran los escenarios o clusters (sec. 27.10 en la pági- na 411) para el área europea con base en el 3 de abril de 2017. Lo que se representa para cada cluster es el miembro del ECENS más próximo al centroide del cluster. Las predicciones entre el D+5 y el D+7 muestran cuatro escenarios con un numero de miembros muy similar distribuidos entre los 4 escenarios. Estos 4 escenarios pueden en realidad reducirse a dos opciones, a saber, bloqueo anticiclónico sobre la Península o aproximación de una vaguada por el norte peninsular. Esta última es precisamente la opción que sigue el modelo determinista. Las predicciones entre $\mathrm{D}+8 \mathrm{y}$ $\mathrm{D}+10$ reducen los escenarios a tres, nuevamente con una distribución parecida de miembros. De nuevo el modelo determinista apoya al cluster con la versión más pesimista en términos de precipitación para la Península. 


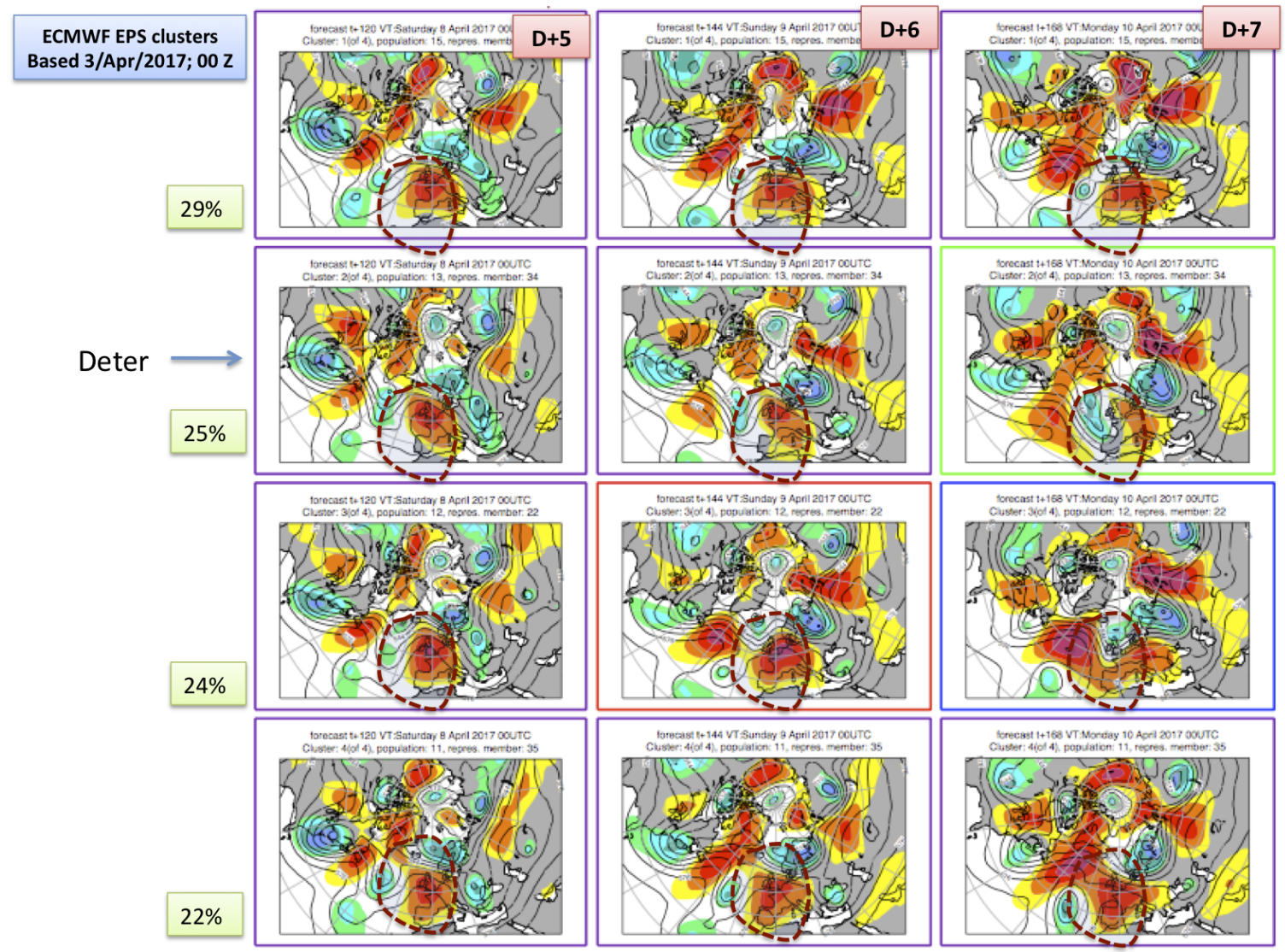

Figura 58.5: Grupos para el área europea con base en el 3 de abril de 2017, D+5..D+7 (ver texto).

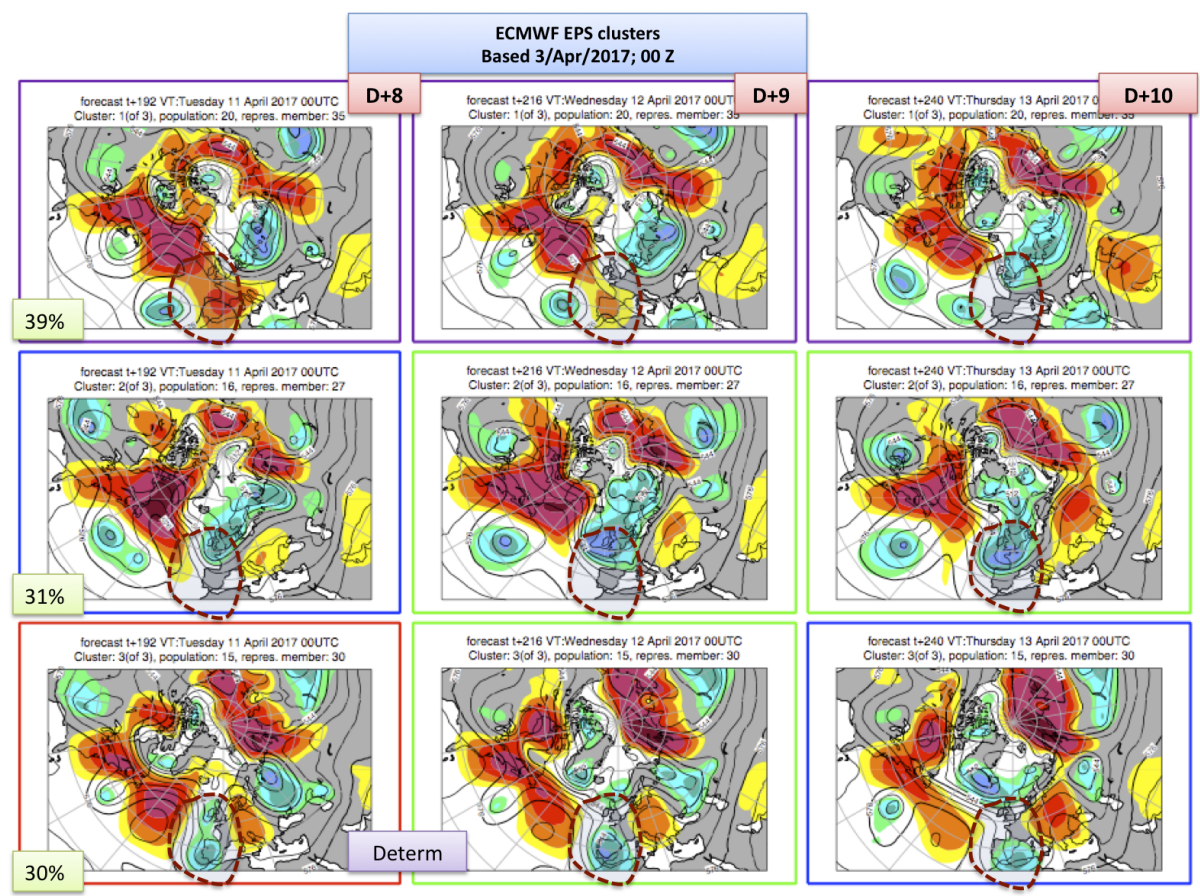

Figura 58.6: Grupos para el área europea con base en el 3 de abril de 2017, D+8..D+10 (ver texto). 

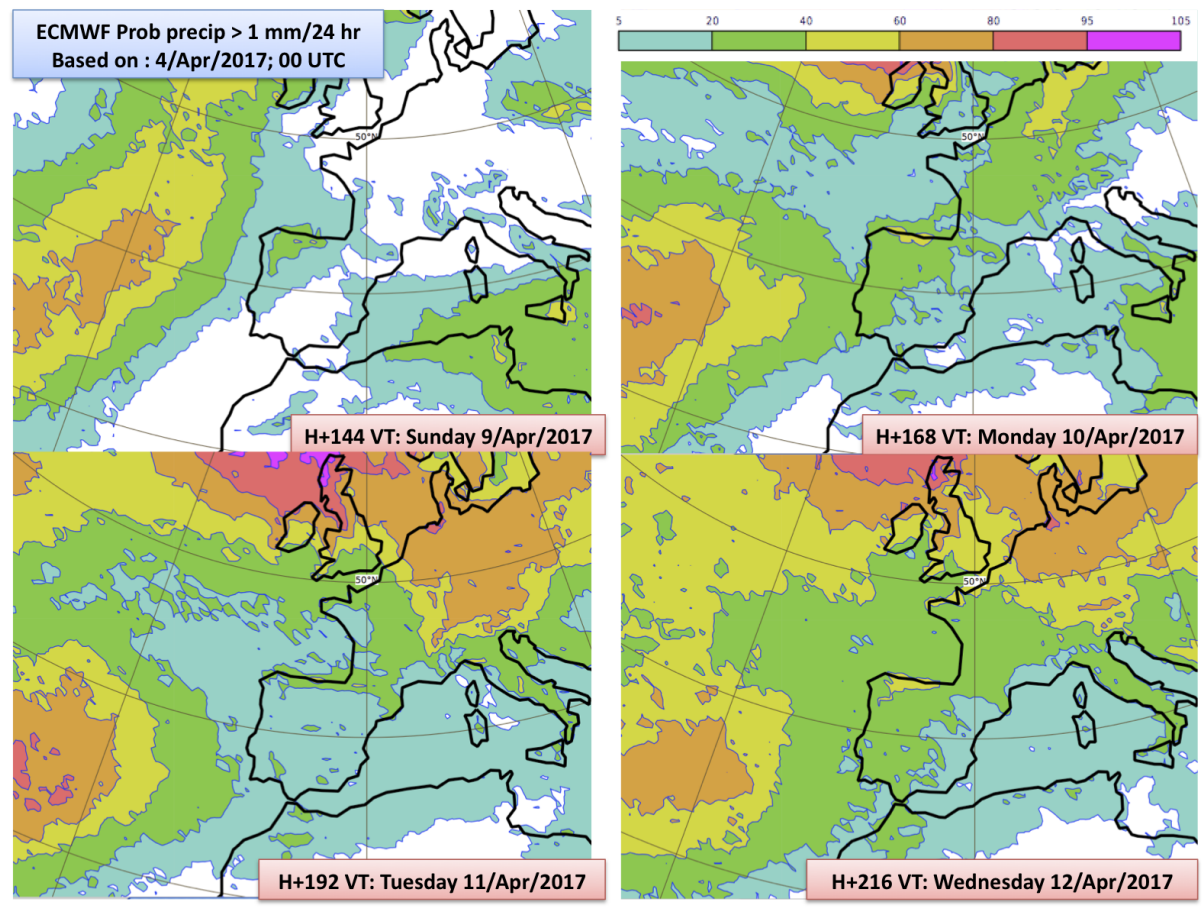

Figura 58.7: Probabilidades ECENS de precipitación superior a $1 \mathrm{~mm}$ en 24 horas basadas en el 4 de abril y válidas desde el Domingo de Ramos 9 de abril $(H+144)$ hasta el Miércoles Santo $(H+216)$.

Llega el momento de muestrear las probabilidades (ver sección 27.6.1 en la página 416) sobre los niveles de precipitación y temperatura para varios umbrales. La figura 58.7, muestra las probabilidades de precipitaciones superiores a $1 \mathrm{~mm}$ en 24 horas basadas en el 4 de abril y válidas desde el Domingo de Ramos 9 de abril (H+144) hasta el Miércoles Santo (H+216). Las probabilidades no son extremas pero sí indican una tendencia a incrementarse en el oeste peninsular a mitad de la semana.

Las predicciones a partir del día 5 de abril, comienzan a apuntar hacia una configuración omega con el aislamiento de una DANA en el Atlántico central y oriental, una dorsal clara sobre la Península y otra DANA sobre el Mediterráneo central. La Figura 58.8 muestra mapas de EM, dispersión y espaguetis basadas en el 5 de abril (izquierda) y 7 de abril (derecha), ambas con validez para los días 11 y 12 de abril.
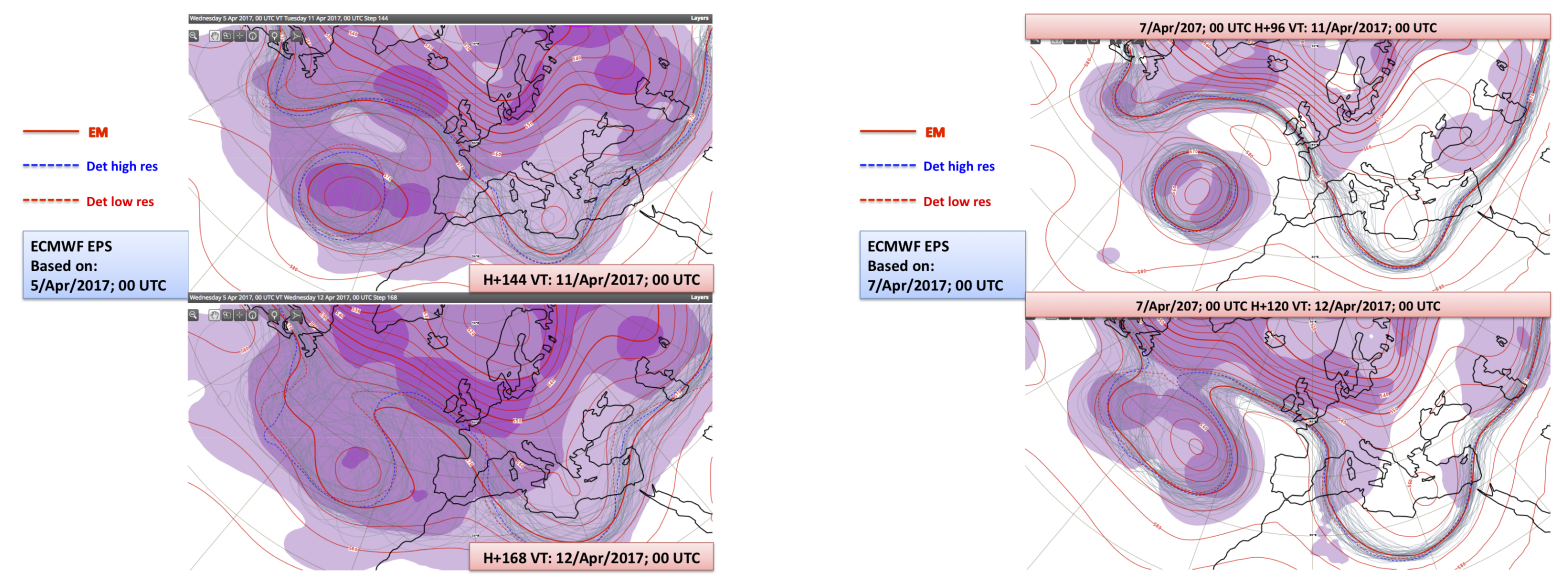

Figura 58.8: Promedio del ECENS, dispersión y espaguetis con validez para los días 11 y 12 de abril. Izquierda: basadas en el 5 de abril. Derecha: basadas en el 7 de abril. 


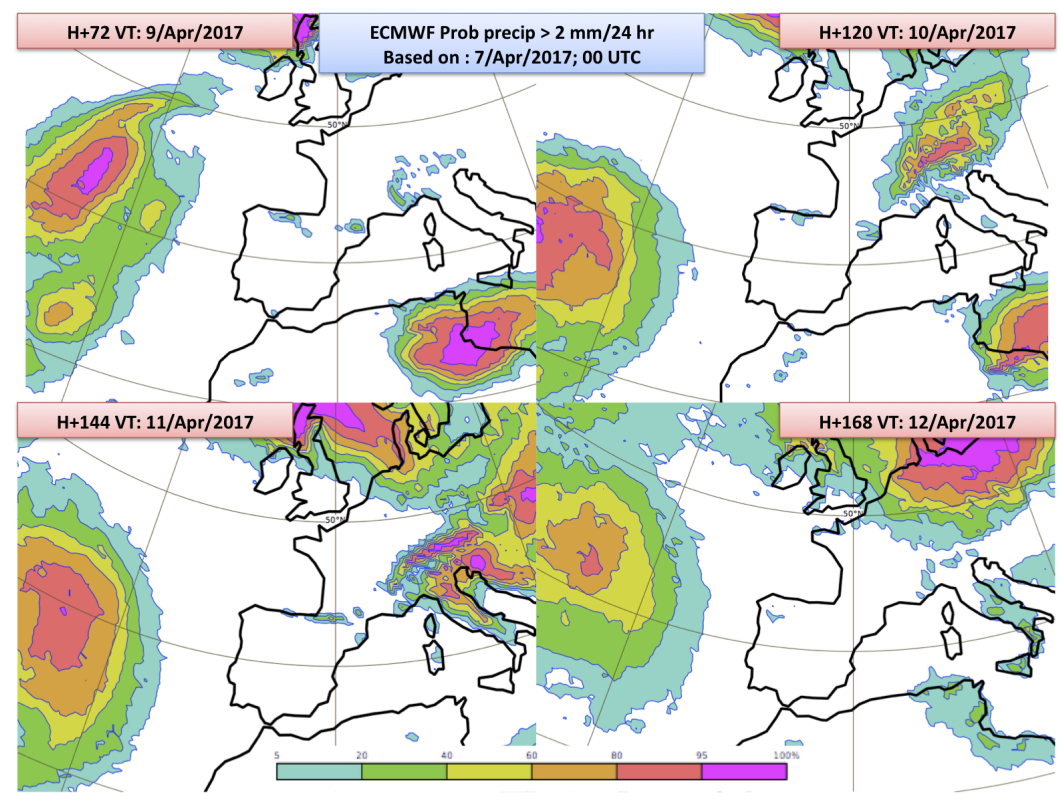

Figura 58.9: Probabilidades ECENS de precipitación superior a 2 mm en 24 horas para el periodo desde el Domingo de Ramos y el Miércoles Santo basadas en el 7 de abril.

Las probabilidades de precipitación superior a $2 \mathrm{~mm}$ en 24 horas que muestra la Figura 58.9 para el periodo desde el Domingo de Ramos y el Miércoles Santo basadas en el 7 de abril señalan un panorama claramente optimista para el sector turístico y lúdico en general. Es más, las predicciones basadas en el 10 de abril (Figura 58.10) muestran altas probabilidades de temperaturas máximas excediendo los 28 grados en el suroeste peninsular. Esto se refleja en el EFI (sección 27.7.2 en la página 420) y el SOT90 (Shift of Tails para el percentil 90 del clima, sección 27.7.3 en la página 421) con valores significativos en todo el cuadrante suroccidental peninsular para el 12 de abril y extendiéndose hacia el norte y este peninsulares el día siguiente.

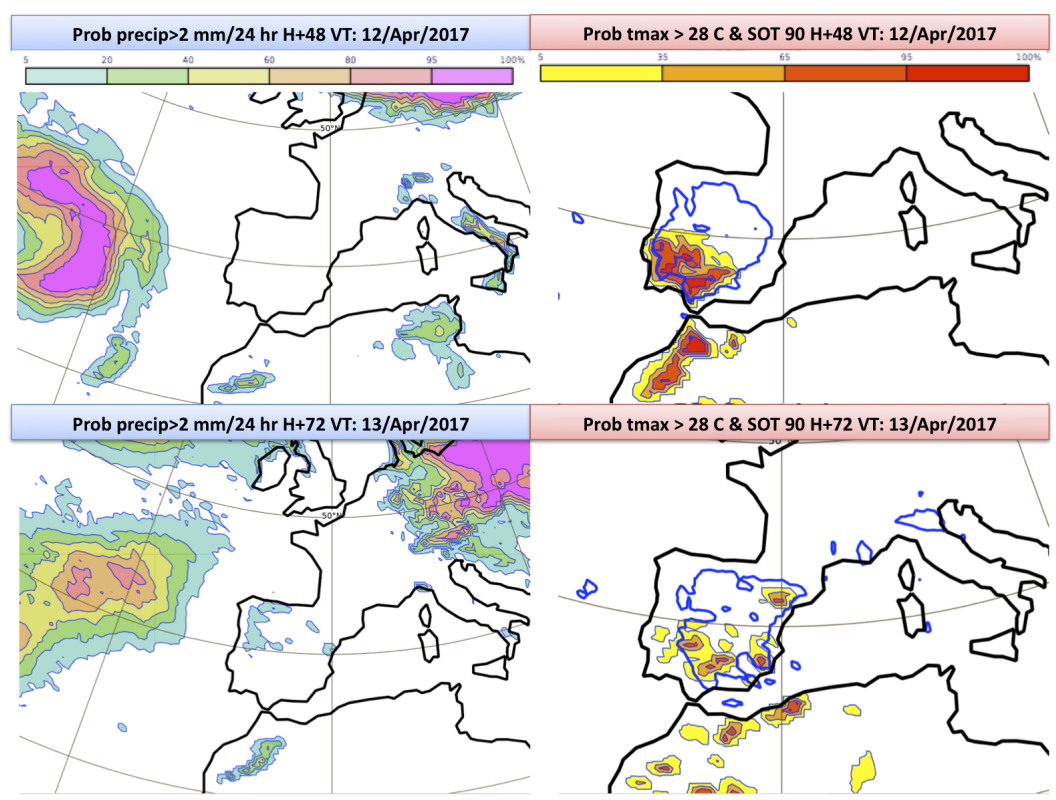

Figura 58.10: Probabilidades ECENS de precipitación superior a $2 \mathrm{~mm}$ en 24 horas y de temperatura superior a 28 grados para el periodo desde el Domingo de Ramos y el Miércoles Santo basadas en el 7 de abril. 
Madrid H+48 VT: 13/Apr/2017

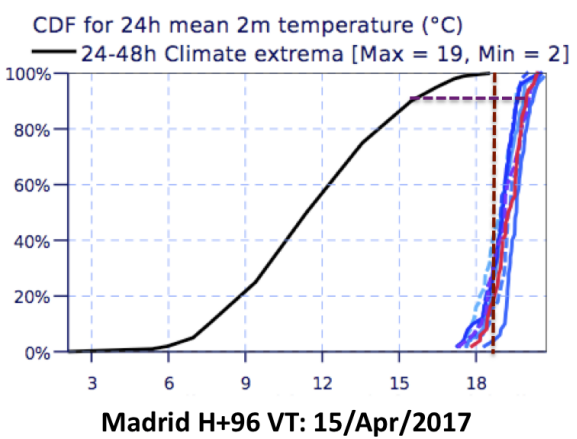

$\mathrm{CDF}$ for $24 \mathrm{~h}$ mean $2 \mathrm{~m}$ temperature $\left({ }^{\circ} \mathrm{C}\right)$

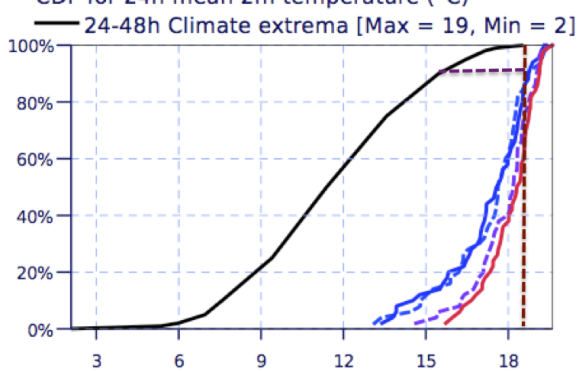

Madrid H+72 VT: 14/Apr/2017

$\mathrm{CDF}$ for $24 \mathrm{~h}$ mean $2 \mathrm{~m}$ temperature $\left({ }^{\circ} \mathrm{C}\right)$

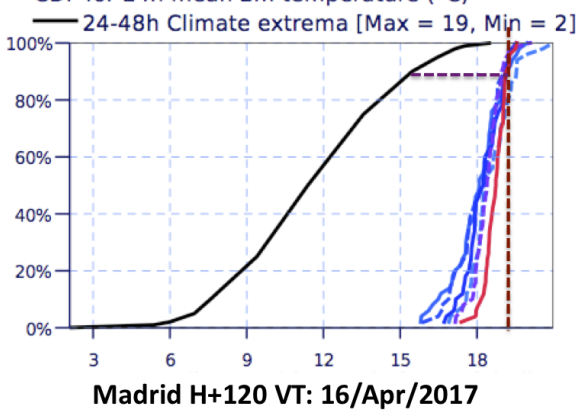

CDF for $24 \mathrm{~h}$ mean $2 \mathrm{~m}$ temperature $\left({ }^{\circ} \mathrm{C}\right)$

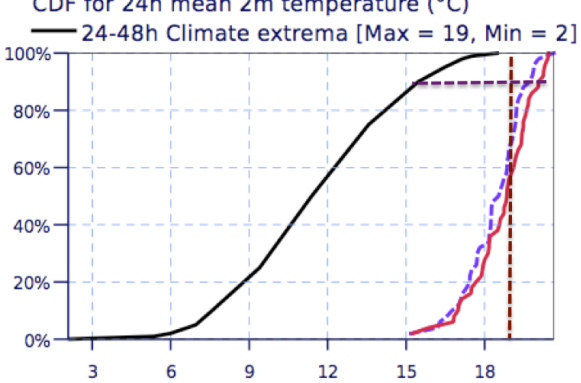

Figura 58.11: CDF (ver texto) de Madrid para la temperatura media a 2 metros basadas en la pasada del 11 de abril de 2017 y con validez para el 13, 14, 15 y 16 de abril $(H+48, H+72, H+96$ y $H+120)$.

Las Figuras 58.11 y 58.12, muestran las CDF (sec. 13 en la página 188) de Madrid y Sevilla para la temperatura media a 2 metros basadas en la pasada del 11 de abril de 2017 y con validez para el 13, 14, 15 y 16 de abril $(\mathrm{H}+48, \mathrm{H}+72, \mathrm{H}+96$ y $\mathrm{H}+120)$. En ambos casos se aprecian las distribuciones de las pasadas sucesivas del ECENS con valores significativamente a la dere- cha de la curva del clima del modelo. Esto apunta a valores altos del EFI. Las pendientes de las distintas pasadas indican también poca dispersión. Cuanto más verticales son estas líneas menor es la dispersión. La presencia de SOT90 superiores a cero también puede apreciarse en estos gráficos.
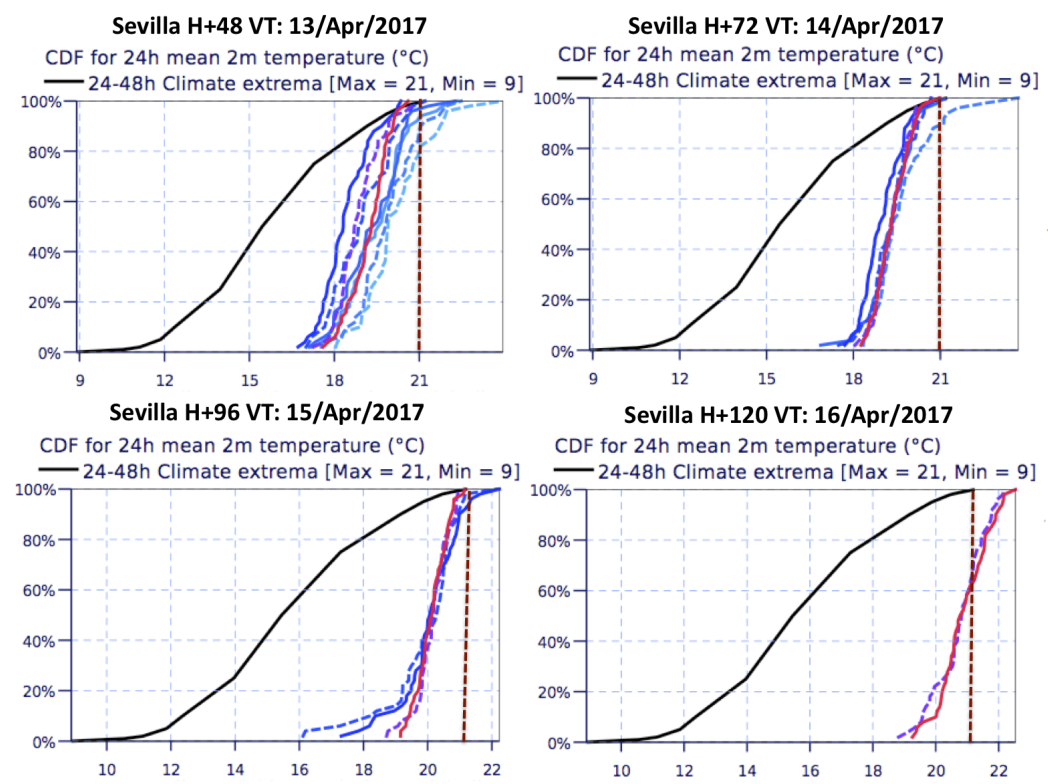

Figura 58.12: CDF (ver texto) de Sevilla para la temperatura media a 2 metros basadas en la pasada del 11 de abril de 2017 y con validez para el 13, 14, 15 y 16 de abril $(H+48, H+72, H+96$ y H+120). 


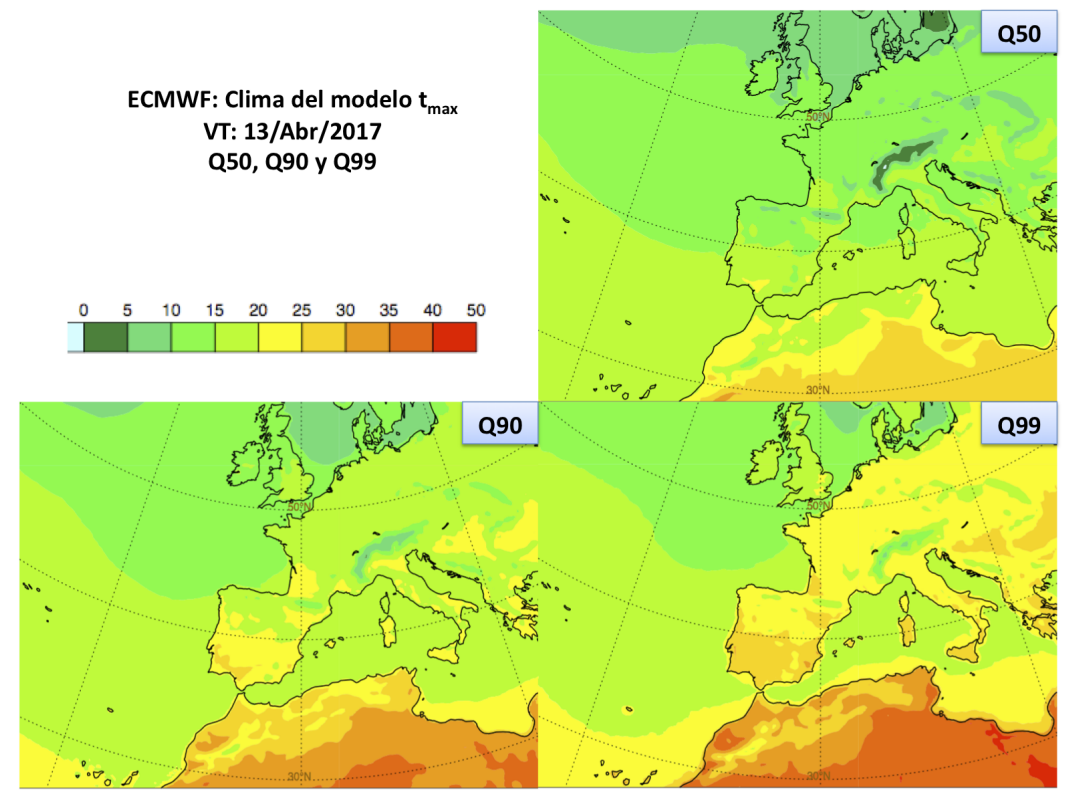

Figura 58.13: Clima del modelo, percentiles 50, 90 y 99, de las temperaturas máximas con validez para el 13 de abril de 2017.

Como referencia, la figura 58.13 muestra el clima del modelo (sección 27.7.1 en la página 419) para las temperaturas máximas con validez para el 13 de abril de 2017. En la figura se muestra la distribución del clima para los percentiles 50, 90 y 99. Aquí puede observarse como, por ejemplo para el percentil 90, valores de Tmax entre 25 y 30 grados sólo aparecen en el valle del Guadalquivir y Extremadura.

\subsection{Datos de observación}

Las Figuras 58.14, 58.15 en la página siguiente, 58.16 y 58.17 en la página 878 muestran datos de observación extraídos de los informes sinópticos de las estaciones de La Coruña, Santander, Sevilla, Málaga, Cáceres, Madrid, Valencia y Palma de Mallorca.

El código surface synoptic observations (SYNOP) se manipula y procesa usando scripts Perl y después utilizando scripts en $R$ para producir los gráficos.

Las series temporales indican claramente la ausencia de precipitaciones (excepto una traza débil en Palma de $0.5 \mathrm{~mm}$ el 16 de abril) y la evolución de las temperaturas en alza comenzando por Andalucía occidental y Extremadura para después extenderse hacia el centro y este peninsular.

\subsection{Resumen final}

El procedimiento realizado ha seguido el método de predicción en cascada.

- La prediccion mensual proporciona una visión general al menos con dos semanas de anticipación (en casos de situaciones extremas hasta tres semanas).

- Para la prediccion a medio plazo el uso de escenarios alternativos y la dispersión de el ECENS nos ayuda a evaluar las distintas opciones a vigilar.

- La utilización de Meteogramas (en particular los que llevan superpuesto el clima del modelo) nos ayuda a evaluar la predicción para diferentes parámetros meteorológicos y localizaciones. La utilización de las distribuciones CDF aportan información muy útil en cuanto a dispersión, EFI y la visualización de valores significativos de SOT en caso de fenómenos extremos.

- El modelo determinista puede considerarse como un miembro más de el ECENS a más resolución.

- Para el corto plazo y la predicción inmediata es donde los Limited Area Model (LAM) de alta resolución, las imágenes de satélite, el radar y la red de descargas juegan un papel determinante. 

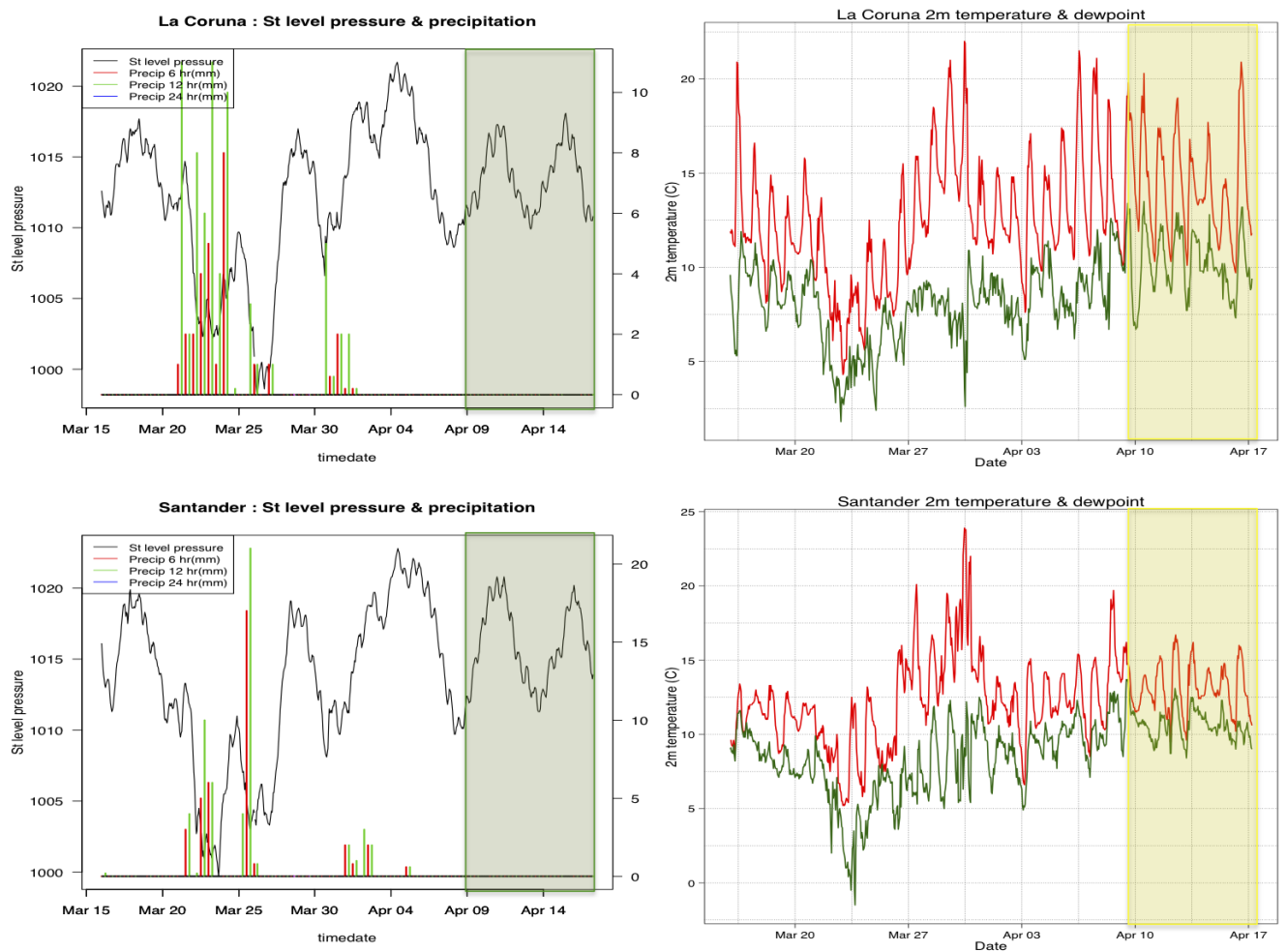

Figura 58.14: Datos de observación extraídos de los informes sinópticos de las estaciones de La Coruña y Santander.
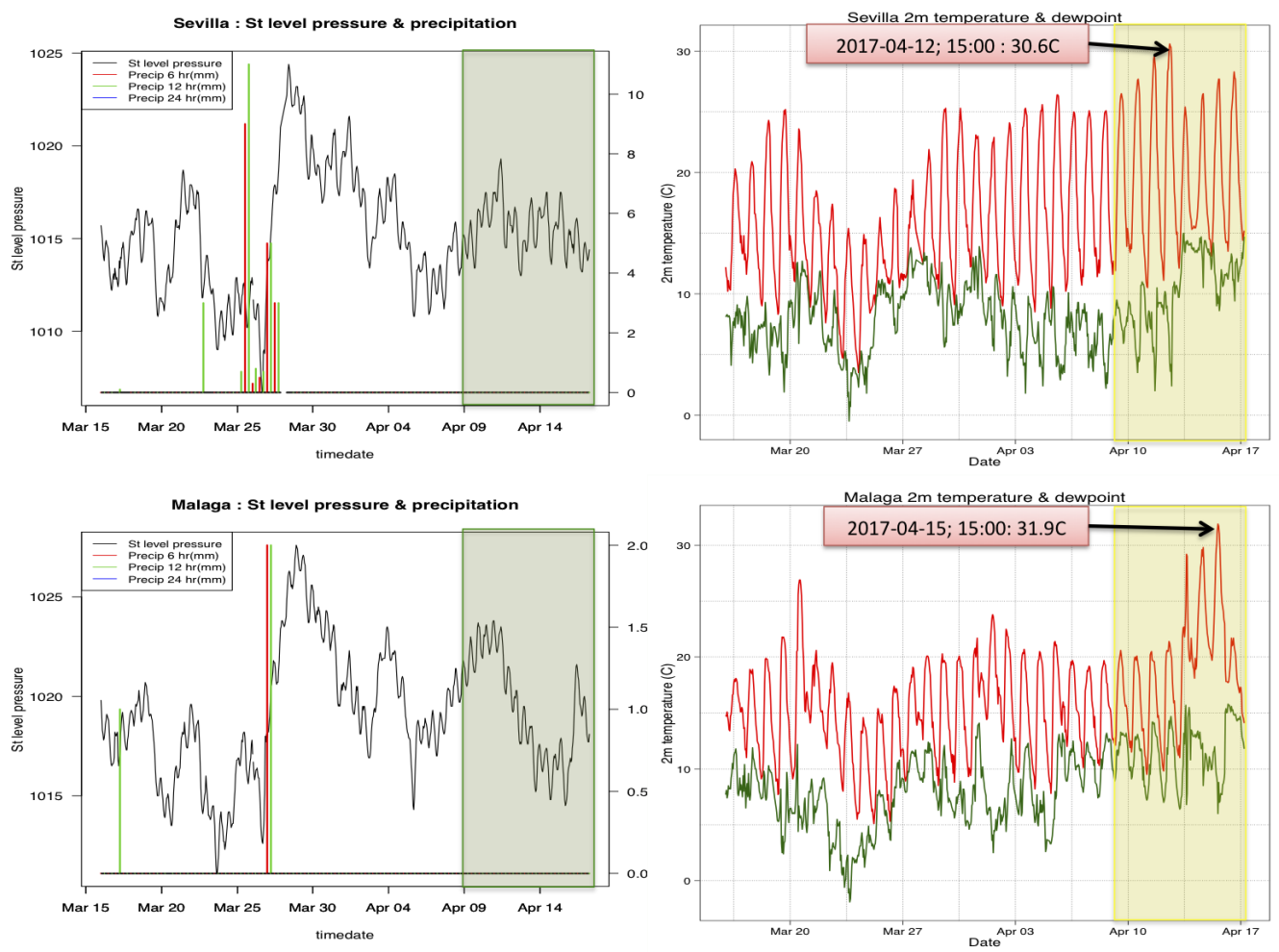

Figura 58.15: Como en la Figura 58.14, ahora Sevilla y Málaga. 

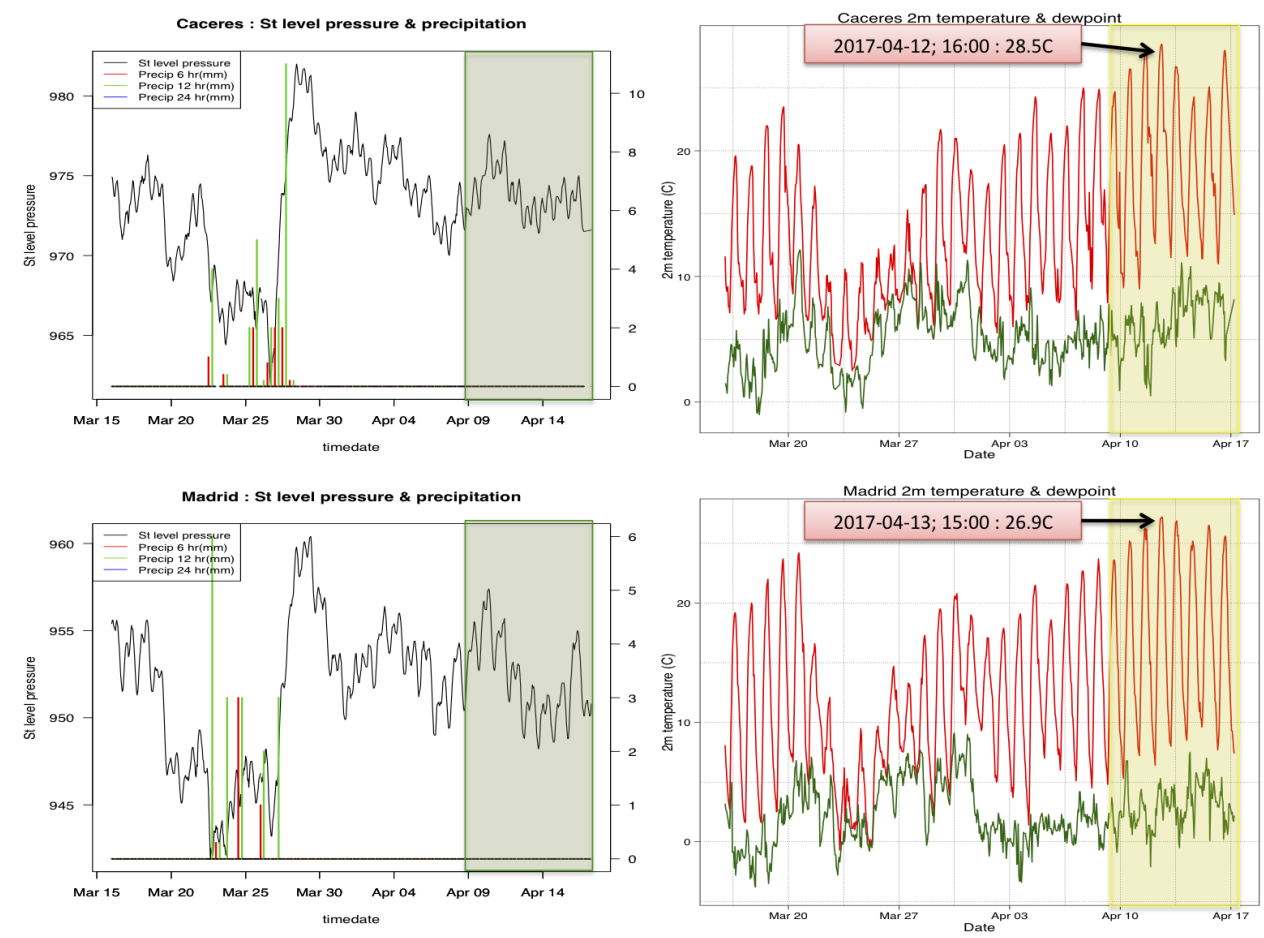

Figura 58.16: Como en la Figura 58.14 en la página anterior, ahora Cáceres y Madrid.
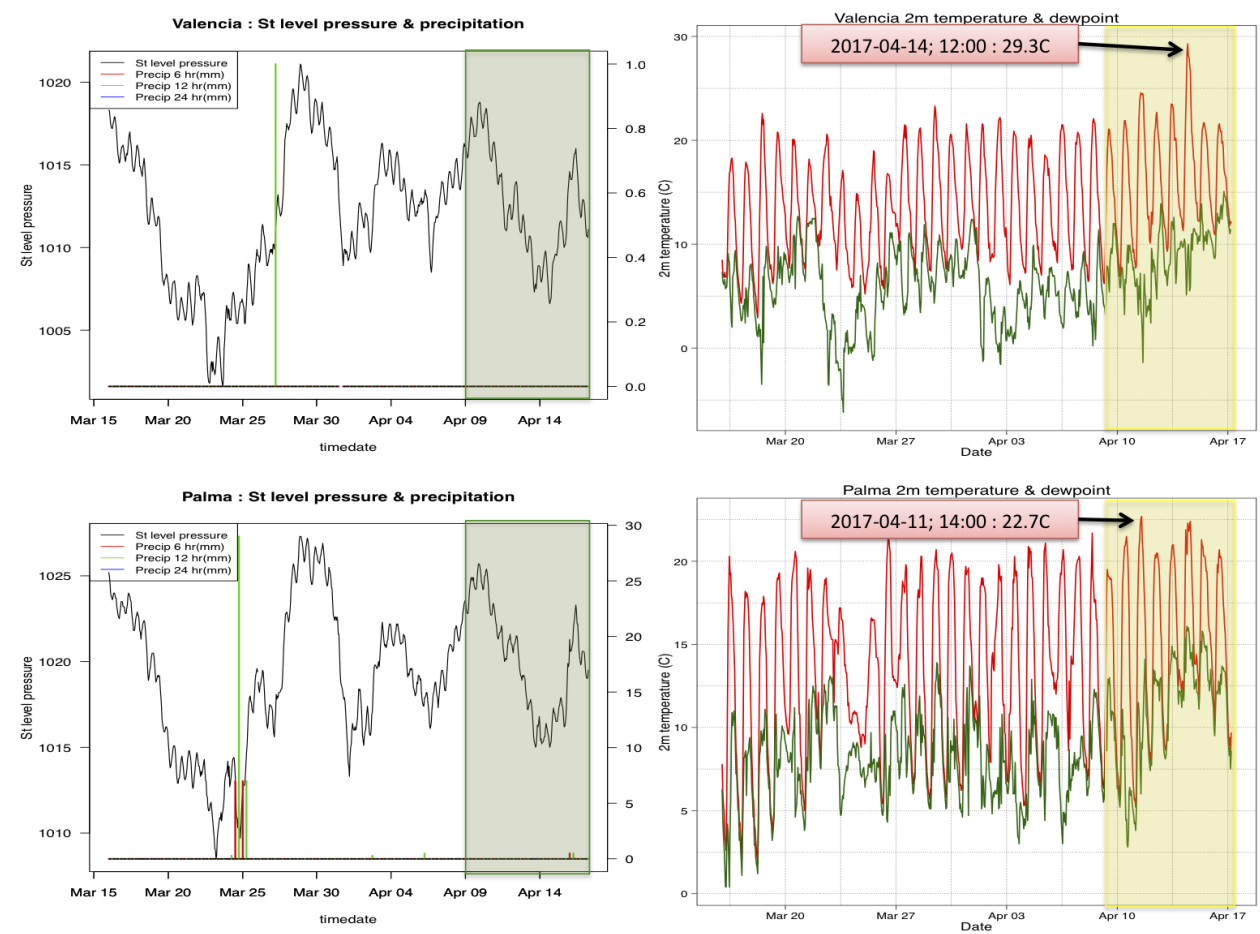

Figura 58.17: Como en la Figura 58.14 en la página anterior, ahora Valencia y Palma de Mallorca. 


\subsection{Referencias}

[1] Arribas, A, Robertson, K B y Mylne, K R. "Test of a poor man's ensemble prediction system for short-range probability forecasting". En: Monthly Weather Review 133.7 (2005), páginas 1825-1839 (citado en página 869$)$.

[2] EBERT, Elizabeth E. y EBERT, Elizabeth E. "Ability of a Poor Man's Ensemble to Predict the Probability and Distribution of Precipitation". En: Monthly Weather Review
129.10 (oct. de 2001), páginas 2461-2480. ISSN: 0027-0644. DOI: 10 . $1175 / 1520-$ 0493 (2001) 129<2461: AOAPMS>2 . 0 . CO; 2 (citado en página 869 ).

[3] Persson, Anders. "User guide to ECMWF forecast products". En: Ecmwf March (2011), página 127 (citado en página 869).

[4] Quiby, J y Denhard, M. "SRNWP-DWD poor-man ensemble prediction system: the PEPS project". En: Eumetnet Newsletter 8 (2003), páginas 9-12 (citado en página 869). 